\title{
Facilitatory Effect of Ventral Cut of Dorsal Raphe Nucleus on Lordosis in Female Rats
}

\author{
MASAKI KAKEYAMA, MAYA NEGISHI, AND KOREHITO YAMANOUCHI \\ Neuroendocrinology, Department of Basic Human Sciences, School of Human Sciences, \\ Waseda University, Saitama 359, Japan
}

\begin{abstract}
The role of the ventral projections of the dorsal raphe nucleus (DRN) in regulating lordosis was examined in female rats. The ventral neural fibers of the DRN were transected by the horizontal circle-cut (VC), anterior (AVC) or posterior half-circle cut (PVC) at the same time as ovariectomy. Three weeks after the surgery, all the rats were implanted with a Silastic tube containing estradiol (E2). The behavioral test was started one day after E2-implantation, and 5 tests in total were carried out every other day. The results showed that the VC females had a higher lordosis quotient (LQ) than control rats. Furthermore, as well as females with the VC, a high score LQ was observed in the AVC rats but not in the PVC females, compared to those in the control animals. These results suggest that the anteroventral neural fibers of the DRN are involved in the lordosis inhibiting mechanisms in female rats.
\end{abstract}

Key words: Dorsal raphe nucleus, Lordosis, Inhibitory system, Cut, Female rat

(Endocrine Journal 44: 589-593, 1997)

FEMALE sexual behavior is regulated by facilitatory and inhibitory mechanisms in the forebrain in rodents [1]. The estrogen-dependent lordosis facilitating influence is thought to exist in the ventromedial hypothalamus, because destruction of it suppresses lordosis, whereas electrical stimulation or direct implantation of crystalline estrogen into the nucleus potentiates the behavior in female rats [see reviews 2 and 3]. In contrast, the lateral septum and the preoptic area exert an inhibitory influence on female sexual behavior, based on the results showing that the destruction of these areas $[4,5]$ or the transection of the fibers of the septum [6] causes a lowering of the estrogen threshold for induction of lordosis. The septum, the preoptic area and the ventromedial

\footnotetext{
Received: September 30, 1996

Accepted: April 7, 1997

Correspondence to: Dr. Korehito YAMANOUCHI, Department of Basic Human Sciences, School of Human Sciences, Waseda University, 2-579-15 Mikajima, Tokorozawa, Saitama 359, Japan
}

hypothalamus send their signals to parts of the lower brainstem, such as the midbrain central gray $[3,7]$ and the pontine periventricular gray $[8,9]$, passing through the medial forebrain bundle [10, 11].

On the other hand, ascending influences from the lower brainstem to the forebrain are also important in regulating lordosis. The serotonergic neuron is concerned with this ascending system. Serotonin is known to play an inhibitory role, because treatment with serotonin antagonist or synthesis-inhibitor lowers the estrogen-threshold to induce lordosis $[12,13]$. Conversely, serotonin agonists suppress lordosis in female rats $[12,13]$.

Most serotonergic neuronal cell bodies are concentrated in the raphe nuclei of the lower brainstem [14]. Notably, the dorsal raphe nucleus (DRN) in the midbrain contains a large number of serotonergic neurons. The DRN sends many their axons to the forebrain including the ventromedial hypothalamus, the septum and the preoptic area $[15,16]$. Destruction of the DRN results in an increase in lordosis activity in not only female but 
also male rats $[17,18]$. This facilitatory effect of DRN lesions is due to damage to serotonergic cell bodies in this nucleus, since serotonin-synthesis inhibitor did not potentiate lordosis in rats with lesions of this nucleus [19]. It can therefore be said that ascending serotonergic neurons of the DRN play an important role in sending lordosis inhibiting signals. Anatomical reports indicate that many efferents of the DRN leave from the ventral side and join the medial forebrain bundle [14, 16]. In this experiment, to elucidate the role of the efferents of the DRN, three types of horizontal cut at the ventral area of the DRN were made and lordosis behavior was examined in estrogen-treated ovariectomized rats.

\section{Materials and Methods}

Female Wistar rats (240-280 g) were housed under a controlled photoperiod (14: $10 \mathrm{~h}$, light: dark) and controlled temperature $\left(23-25^{\circ} \mathrm{C}\right)$. Fortythree females were ovariectomized and thirty were subjected to the brain surgery under ether anesthesia. The horizontal circle-cut (VC) and anterior or posterior half-circle cut (AVC or PVC, respectively) at the ventral area of the DRN was made with a L-shaped Halász knife with a $1.5 \mathrm{~mm}$ blade. Under a stereotaxic instrument with an incisor bar set at $11 \mathrm{~mm}$ below the interaural line, the knife was lowered $5.2 \mathrm{~mm}$ from the bregma level at a point $9.8 \mathrm{~mm}$ posterior to the bregma on the midline (tip of the blade), and rotated $360^{\circ}$ in $11 \mathrm{VC}$ rats, anterohorizontally $180^{\circ}$ in $12 \mathrm{AVC}$ rats and posterohorizontally $180^{\circ}$ in $10 \mathrm{PVC}$ rats (see Fig. 1). In the sham-operated (sham) group, the knife was lowered to the same point as in the VC group and removed without rotation in 10 rats. In addition, 13 ovariectomized female rats without brain surgery were prepared as controls.

Two or 3 weeks after the operations, all females were implanted subcutaneously with a Silastic tube (1.5 cm in length, 1.57: $3.18 \mathrm{~mm}$, I.D.: O.D., DowCorning No. 602-285) containing estradiol-17- $\beta$ (E2; Sigma). The day after E2 implantation, behavioral test was started and a total of 5 tests were carried out every other day for 9 days. In the behavioral test, each female rat was placed in an observation cage with two vigorous males, and the lordosis quotient (LQ; number of lordosis /10 mounts, $x$ 100) was recorded.

At the end of the behavioral test, to determine the precise localization of the cut, histological examination of the brain was performed. The rats were killed by overexposure to ether. The brain of each was removed and fixed in $10 \%$ formalin solution. Subsequently, $100 \mu \mathrm{m}$ frozen sections were made and then stained with cresyl fast violet. The LQs were analyzed by Mann-Whitney U-test (significant when $P<0.05$ ). The data regarding the

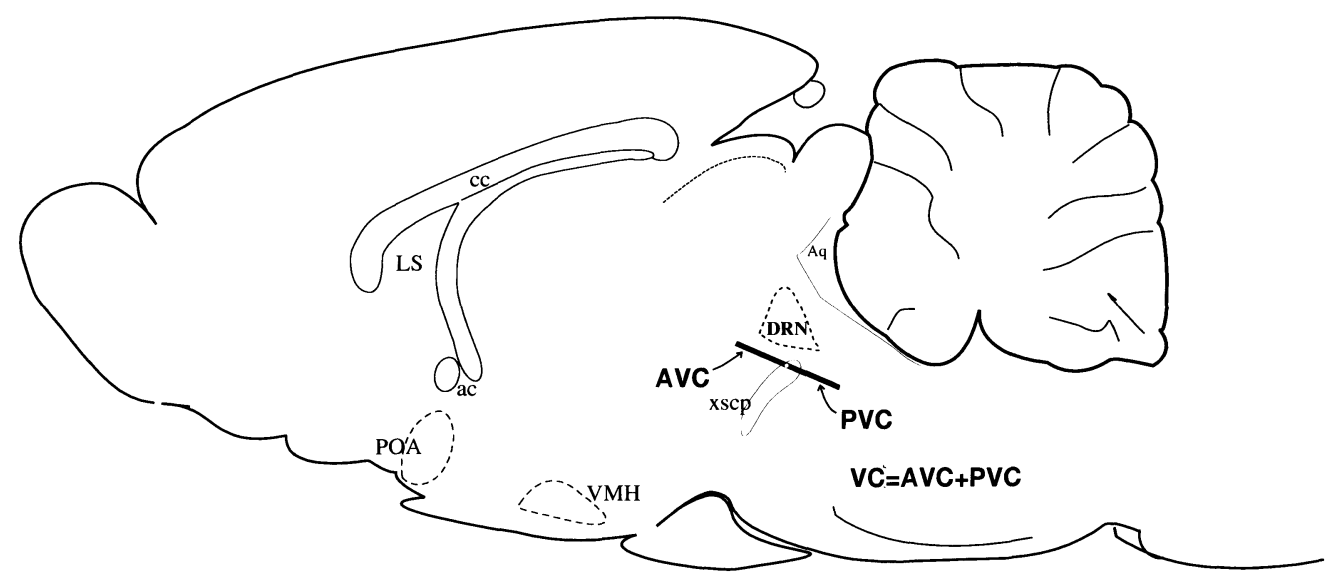

Fig. 1. Schematic representation of the surgical cuts in the midsagittal section of the rat brain. The horizontal black bar just below the dorsal raphe nucleus (DRN) indicates the ventral horizontal cut (VC). The anterior (AVC) and posterior (PVC) horizontal cuts correspond to the anterior and posterior parts of the $\mathrm{VC}$, respectively. ac, anterior commissure; $\mathrm{Aq}$, aqueduct; cc, corpus callosum; LS, lateral septum; POA, preoptic area; $\mathrm{VMH}$, ventromedial hypothalamic nucleus; xscp, decussation of superior cerebellar peduncle. The drawing is modified from the Stereotaxic Atlas of Paxinos and Watson [21]. 
incidence of lordosis were evaluated by means of the Chi-square test.

\section{Results}

In the first test, only 2 out of 13 rats and 2 of 10 rats displayed lordosis in control and sham groups, respectively, and the LQs of these females were $2.3 \pm 1.7$ (mean \pm SEM) and $2.0 \pm 1.3$, respectively (Fig. 2). In contrast, 7 of $11 \mathrm{VC}$ females displayed lordosis $(P<0.05, v s$. control and sham groups) and the mean $L Q$ was $18.2 \pm 4.6$, being significantly higher than those in the control $(P<0.05)$ and sham $(P<0.05)$ groups. In AVC group, 8 of 12 rats displayed lordosis, the mean LQ $(25.8 \pm 8.7)$ being significantly higher than those in the control $(P<0.05)$ and sham $(P<0.05)$ groups. In the PVC group, none of the rats showed lordosis behavior in the first test. In all the groups, an increase in incidence and the mean LQ was observed throughout 5 behavioral tests. In the last test, the mean LQs of the control and sham groups were $70.8 \pm 8.4$ and $68.0 \pm 9.5$, respectively. In the VC group, the mean LQ $(93.6 \pm 6.4)$ was still higher than in the control $(P<0.05)$ and the sham $(P<0.05)$ groups. The mean LQ $(92.5 \pm 6.6)$ in AVC females was also significantly higher than those in the sham animals $(P<0.05)$. In the PVC group, the mean LQ was $80.0 \pm 7.59$, being comparable to those in control and sham groups.

There were no significant differences in body weight among groups. The locomotor activity in females with the cuts did not seem to differ from that in the control rats, although systematic analyses were not performed in this experiment.

The localization of the cut was determined according to the rat brain atlas of Paxinos and Watson [20]. Most VC were located just above the decussation of the superior cerebellar peduncle. The extension of the VC in the anteroposterior axis is from the level of the posterior part of the oculomotor nuclei to the level of the dorsal tegmental nucleus. The AVC and PVC also were located at the same level as the VC in the dorsoventral axis. The cuts extended from the level of the dorsal part of the superior cerebellar peduncle decussation to the level of oculomotor nuclei in the AVC group or to the dorsal tegmental nucleus in the PVC group in the anteroposterior axis.

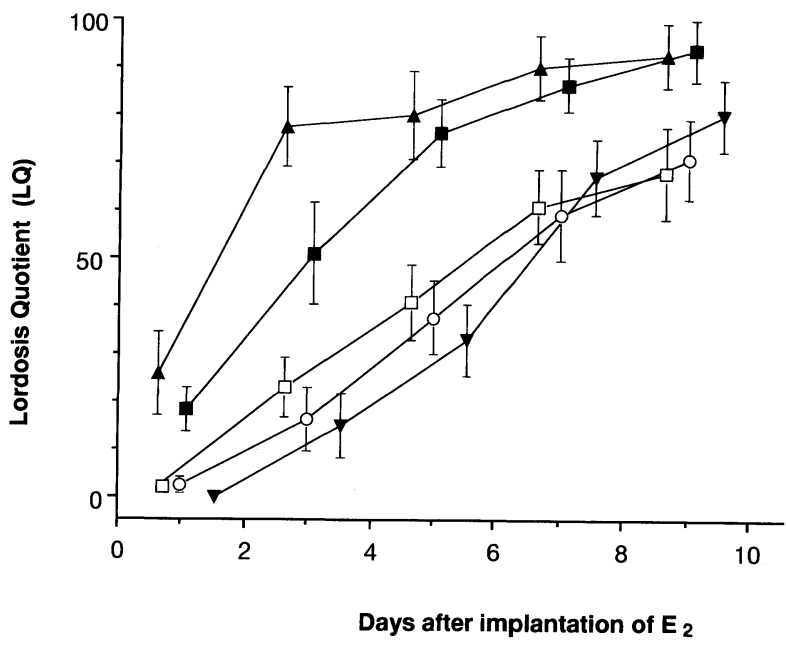

Fig. 2. The mean lordosis quotients (LQs) in female rats with a ventral(VC), anterior ventral (AVC) or posterior ventral (PVC) cut in the dorsal raphe nucleus. Behavioral tests began from one day after implantation of a Silastic tube containing estradiol and were carried out for a total of 5 times every other day. $\bigcirc$, control (13), $\square$, sham (10); $\square$, VC (11), $\Delta, \operatorname{AVC}(12), \nabla, \operatorname{PVC}(10)$.

\section{Discussion}

In the present experiment, $\mathrm{VC}$ and AVC potentiated lordosis behavior in estrogen-treated ovariectomized rats. The DRN plays an inhibitory role in mediating lordosis behavior in female and male rats $[17,18]$. The inhibition is thought to be produced by serotonergic neurons in the DRN [19]. A large number of serotonergic terminals of the DRN have been verified in the forebrain and send their axons to the forebrain $[15,16]$. From these reports, it can be said that the facilitatory effect of the VC and the AVC on lordosis in this results can be attributed to the cut of the ascending serotonergic efferent fibers of the DRN.

It has been reported that the distribution of the projections of the rostral DRN in the forebrain is not always same as that of the caudal DRN [21]. The rostral DRN sends axons to the preoptic area, the lateral and caudal hypothalamus. Many axons of the caudal part of the DRN terminate at the bed nucleus of the stria terminalis, the caudate, the hippocampus, the septum and the preoptic area.

The ventromedial hypothalamus is known to exert a lordosis facilitating influence [2]. Since implantation of a toxin specific to serotonergic 
neurons into the ventromedial hypothalamus facilitates lordosis [22], it is possible to say that the serotonergic fibers which leave the anteroventral side of the rostral DRN modify the function of lordosis-facilitating mechanisms of the ventromedial hypothalamus. The septal area which plays an inhibitory role in regulation of lordosis [23] also receives serotonergic axons of the DRN [15, 21]. However, serotonin agonists, such as ergocornine [24] and p-chloramphetamine [5] suppress lordosis in female rats, even after the septal outputs are interrupted. Although the septal area mainly receives neural projections from the caudal part of the DRN [21], the PVC which interrupts the posterior outputs had no effect on lordosis in the present experiment. Based on these reports, the septal area can be excluded from the possible focus of the lordosis inhibiting signals from the DRN. As the septum, the preoptic area exerts a lordosis inhibiting influence [23]. This area receives many projections from both rostral and caudal DRN [21]. The preoptic area is therefore one of possible sites of action of the rostral DRN serotonergic neurons in the lordosis regulating system.

Further experiments are needed to clarify the site of action of the anteroventral serotonergic efferent fibers of the rostral DRN in the forebrain.

\section{Acknowledgement}

We wish to express our hearty thanks to Mr. F. Maekawa and Mr. T. Matsumoto of Waseda University for their help with the statistical analysis and preparation of figures. This study was supported by Grants-in-Aid from the Ministry of Education, Science and Culture of Japan to M. K. and K. Y. and a Research Grant from Waseda University (97A-368) to K. Y.

\section{References}

1. Yamanouchi K (1980) Inhibitory and facilitatory neural mechanisms involved in the regulation of lordosis behavior in female rats: Effects of dual cuts in the preoptic area and hypothalamus. Physiol Behav 25: 721-725.

2. Pfaff DW, Schwartz-Giblin S, McCarthy MM (1994) Cellular and molecular mechanisms of female reproductive behaviors. In: Knobil E, Neill J (eds) The Physiology of Reproduction. 2nd ed, Raven, New York, 2: 107-220.

3. Sakuma Y (1992) Brain control of female sexual behavior. In: Yokoyama A (ed) Brain Control of the Reproductive System. Japan Sci. Soc. Press/CRC Press, Tokyo: 141-163.

4. Nance DW, Shryne J, Gorski RA (1975) Effects of septal lesions on behavioral sensitivity of female rats to gonadal hormones. Horm Behav 6: 59-64.

5. Yamanouchi K, Watanabe H, Okada R, Arai Y (1982) Forebrain lordosis inhibiting system and serotonin neuron in female rats: Effects of p-chloroamphetamine. Endocrinol Japon 29: 469-474.

6. Yamanouchi K, Arai Y (1977) Possible inhibitory role of the dorsal inputs to the preoptic area and hypothalamus in regulating female sexual behavior in the female rat. Brain Res 127: 296-301.

7. Kondo Y, Koizumi T, Arai Y, Kakeyama M, Yamanouchi K (1993) Functional relationships between mesencephalic central gray and septum in regulating lordosis in female rats: Effect of dual lesions. Physiol Behav 32: 635-638.

8. Hennessey AC, Camak L, Gordon F, Edwards DA (1990) Connections between the pontine central gray and the ventromedial hypothalamus are essential for lordosis in female rats. Behav Neurosci 104: 477488.

9. Yamanouchi K, Arai Y (1983) Forebrain and lower brainstem participation in facilitatory and inhibitory regulation of the display of lordosis in female rats. Physiol Behav 30: 155-159.

10. Modianos DT, Delia H, Pfaff DW (1976) Lordosis in female rats following medial forebrain bundle lesions. Behav Biol 18: 135-141.

11. Yamanouchi K, Arai Y (1989) Lordosis-inhibiting pathway in the lateral hypothalamus: Medial forebrain bundle (MFB) transection. Zool Sci 6: 141145.

12. Meyerson BJ, Malmnäs C-O (1978) Brain monoamines and sexual behaviour. In: Hutchison JB (ed) Biological Determinants of Sexual Behaviour. Wiley, New York: 521-554.

13. Zemlan FP, Ward IL, Crowley WR, Margules DL (1973) Activation of lordotic responding in female rats by suppression of serotonergic activity. Science 179: 1010-1011.

14. Törk I (1985) Raphe nuclei and serotonin containing systems. In: Paxinos G (ed) The Rat Nervous System. Hindbrain and spinal cord. Academic Press, New York 2: 43-78. 
15. Azmitia EC, Segel M (1978) An autoradiographic analysis of the differential ascending projections of the dorsal and median raphe nuclei in the rat. $J$ Comp Neurol 179: 641-668.

16. Parent A, Descarries L, Beaudet A (1981) Organization of ascending serotonin systems in the adult rat brain. A radioautographic study after intraventricular administration of [3H]5hydroxytryptamine. Neurosci 6: 115-138.

17. Yamanouchi K, Arai Y (1985) The role of mesencephalic tegmentum in regulating female rat sexual behaviors. Physiol Behav 35: 393-259.

18. Kakeyama M, Yamanouchi K (1992) Lordosis in male rats: The facilitatory effect of dorsal raphe nucleus lesion. Physiol Behav 51: 181-184.

19. Kakeyama M, Yamanouchi K (1993) Female sexual behaviors in male rats with dorsal raphe nucleus lesions: Treatment with p-chlorophenylalanine. Brain Res Bull 30: 705-709.

20. Paxinos G, Watson C (1986) The Rat Brain in
Stereotaxic Coordinates. 2nd ed, Academic Press, New York.

21. Vertes RP (1991) A PHA-L analysis of ascending projections of the dorsal raphe nucleus in the rat. $J$ Comp Neurol 313: 643-668.

22. Luine VN, Frankfurt M, Rainbow TC, Biegon A, Azmitia E (1983) Intrahypothalamic 5,7dihydroxytryptamine facilitates feminine sexual behavior and decrease [3H]-imipramine binding and 5-HT uptake. Brain Res 264: 344-348.

23. Yamanouchi K, Matsumoto A, Arai Y (1985) Neural and hormonal control of lordosis behavior in the rat. Zool Sci 2: 617-627.

24. Arai Y, Yamanouchi K (1979) Inhibition of lordosis by ergocornine in estrogen-progesterone primed ovariectomized rats. In: Ito M, Tsukahara N, Kubota K, Yagi K (eds) Integrative Control Functions of the Brain. Kodansha, Tokyo/ Elsevier/Amsterdam 2: 353-355. 